\title{
Pacific
}

Journal of

Mathematics

\section{ON POLYNOMIALS ORTHOGONAL WITH RESPECT TO SOBOLEV INNER PRODUCT ON THE UNIT CIRCLE}

Xin Li AND Francisco MARCELlan 


\title{
ON POLYNOMIALS ORTHOGONAL WITH RESPECT TO SOBOLEV INNER PRODUCT ON THE UNIT CIRCLE
}

\author{
Xin Li and Francisco Marcellan
}

\begin{abstract}
We study polynomials orthogonal with respect to an indefinite Sobolev inner product on the unit circle. We establish the existence of such polynomials of large degree. Algebraic properties and asymptotic behavior of such polynomials are obtained.
\end{abstract}

\section{Introduction.}

The study of orthogonal polynomial with respect to standard inner product, i.e.

$$
\langle f, g\rangle=\int_{\Gamma} f(z) \overline{g(z)} d \mu(z)
$$

where $\Gamma$ is a curve in the complex plane and $\mu$ is a positive definite Borel measure supported on $\Gamma$, constitutes an important subject of research in several areas such as approximation theory, numerical analysis as well as in other applied fields (signal processing, linear systems, ets.).

Recently, many people have been interested in the analysis of orthogonal polynomials with respect to some nonstandard inner products. One of the most important examples is related with Sobolev inner products.

In particular, much attention is now been paid to the case

$$
\langle f, g\rangle=\int_{I} f(x) g(x) d \mu_{0}(x)+\int_{J} f^{\prime}(x) g^{\prime}(x) d \mu_{1}(x),
$$

where $\mu_{0}$ and $\mu_{1}$ are positive definite Borel measures supported on $I$ and $J$, subsets of the real line, respectively (see [10] for a survey of the subject matter). But very few results are known when the support of the measures is not contained in the real line.

A study of Borel measures supported on the unit circle has been initiated in $[\mathbf{3}]$, where the case when the inner product is given by

$$
\langle f, g\rangle=\int_{|z|=1} f(z) \overline{g(z)} d \mu+\lambda f^{\prime}(a) \overline{g^{\prime}(a)}
$$


where $\mu$ is a Borel measure on $|z|=1$ and $|a|=1$, is investigated and some questions such as representation formulas and relative asymptotics for the new sequence of orthogonal polynomial with respect to the standard one are provided. A comparison with the usual applications of the standard orthogonal polynomials, problems such as the location of zeroes, quadrature formulas, relation with continued fractions, and rational approximation to some integral transforms of measures need further studies.

Our present work can be focussed from two different points of view:

First, we perturb a standard inner product as in (1) on the unit circle using the first derivatives in several points off the circle instead of using higher order derivatives at only one point as in the direction pointed out in some recent research when the support of measure is contained in the real line (cf. $[\mathbf{1}, \mathbf{8}, \mathbf{1 1}])$.

Secondly, we drop the requirement of positivity and Hermitian character of the inner product and establish the existence of orthogonal polynomials of large degree. When the measure $\mu$ belongs to a wide class $N$ (the analogue of Nevai's class, see the definition in $\S 3$ ), we obtain the relative asymptotics of the two families of orthogonal polynomials associated to the measure $\mu$ and the nonstandard inner product, respectively. These extend the results in $[\mathbf{3}]$.

The organization of the paper is as follows: $\S 1$ is devoted to some definitions and basic facts; $\S 2$ collects some algebraic properties: $\S 3$ contains the statement of our main results on relative asymptotics whose proofs are then given in $\S 4$.

\section{Notations.}

Let $d \mu$ be a positive measure on the unit circle $|z|=1$ with an infinite set of support. Let $z_{1}, z_{2}, \ldots, z_{m}$ be $m$ fixed points in the complex plane $\mathbb{C}$. Additional assumptions on the location of $\left\{z_{i}\right\}$ will be made later. Throughout this paper, $\boldsymbol{Z}$ denotes the vector $\left(z_{1}, z_{2}, \ldots, z_{m}\right)$. We will use $\mathcal{P}_{n}$ to denote the set of polynomials of degree at most $n$ with complex coefficients.

Let $\varphi_{n}(z)$ be the $n$-th orthonormal polynomial associated with $d \mu$, i.e., $\varphi_{n}(z)=\kappa_{n} z^{n}+\cdots \in \mathcal{P}_{n}$ with $\kappa_{n}>0$ and

$$
\int \varphi_{n}(z) \overline{z^{k}} d \mu=\frac{\delta_{n, k}}{\kappa_{n}}, k=0,1, \ldots, n
$$

It is convenient to apply the following convention: For any function $F(z)$ of a single variable $z$, we write $F(\boldsymbol{Z})$ for the vector $\left(F\left(z_{1}\right), F\left(z_{2}\right), \ldots, F\left(z_{m}\right)\right)$. 
So we have, for example,

$$
\begin{aligned}
& f^{\prime}(\boldsymbol{Z})=\left(f^{\prime}\left(z_{1}\right), f^{\prime}\left(z_{2}\right), \ldots, f^{\prime}\left(z_{m}\right)\right) \text { and } \\
& K(z, \boldsymbol{Z})=\left(K\left(z, z_{1}\right), K\left(z, z_{2}\right), \ldots, K\left(z, z_{m}\right)\right),
\end{aligned}
$$

if $f(z)$ is differentiable and $K(z, w)$ is a function of two variables $z$ and $w$. Define an indefinite inner product as follows

$$
\langle f, g\rangle:=\int f \bar{g} d \mu+f^{\prime}(\boldsymbol{Z}) \boldsymbol{A} g^{\prime}(\boldsymbol{Z})^{H},
$$

where $\boldsymbol{v}^{H}$ denotes the conjugate transpose of a vector $\boldsymbol{v}$, and $\boldsymbol{A}$ is a $m \times m$ complex matrix. Throughout this paper, matrices will always be denoted by bold-face letters.

We say a polynomial $\psi_{n}(z) \in \mathcal{P}_{n}$ is (left-) orthonormal with respect to the indefinite inner product $\langle\cdot, \cdot\rangle$ if

$$
\left\langle\psi_{n}, z^{k}\right\rangle=0, k=0,1, \ldots, n-1,
$$

and

$$
\left|\left\langle\psi_{n}, \psi_{n}\right\rangle\right|=1
$$

Cf. [2]. If $\boldsymbol{A}$ is a Hermitian positive-definite matrix, then the existence and uniqueness of such polynomials is always guaranteed. In general, we can see that if such a polynomial exists then $\operatorname{deg} \psi_{n}=n$, and in this case we will always assume the leading coefficient of $\psi_{n}$ is positive and denoted by $\gamma_{n}$. Even with these conventions, the uniqueness of $\psi_{n}$ is still unknown; nevertheless, under the assumption that $\boldsymbol{A}$ is non-singular, we will show that such polynomials exist for $n$ sufficiently large. In the sequel, we will use $\psi_{n}$ to denote one of such polynomials. The theory is a natural extention of the results for the real line (cf. [8]).

\section{Algebraic Properties.}

We list some useful relations between $\left\{\varphi_{n}(z)\right\}$ and $\left\{\psi_{n}(z)\right\}$. Let

$$
K_{n}(z, \zeta)=\sum_{j=0}^{n-1} \varphi_{j}(z) \overline{\varphi_{j}(\zeta)}
$$

Then $K_{n}$ is the reproducing kernel in $\mathcal{P}_{n-1}:$ for $f \in \mathcal{P}_{n-1}$

$$
\int f(\zeta) K_{n}(z, \zeta) d \mu(\zeta)=f(z)
$$


Put

$$
K_{n}^{(i, j)}(z, \zeta)=\sum_{k=0}^{n-1} \varphi_{k}^{(i)}(z) \overline{\varphi_{k}^{(j)}(\zeta)}, i, j=0,1,2, \ldots
$$

Then for $f \in \mathcal{P}_{n-1}$,

$$
\int f(\zeta) K_{n}^{(i, 0)}(z, \zeta) d \mu(\zeta)=f^{(i)}(z)
$$

Note also that $K_{n}^{(i, j)}(z, \zeta)=\overline{K_{n}^{(j, i)}(\zeta, z)}$.

Formula 1. If $\psi_{n}$ exists, then

$$
\psi_{n}(z)=\frac{\gamma_{n}}{\kappa_{n}} \varphi_{n}(z)-\psi_{n}^{\prime}(\boldsymbol{Z}) \boldsymbol{A} K_{n}^{(0,1)}(z, \boldsymbol{Z})^{t},
$$

where $\boldsymbol{v}^{t}$ denotes the transpose of a vector $\boldsymbol{v}$.

Proof. Note that

$$
\psi_{n}(z)-\frac{\gamma_{n}}{\kappa_{n}} \varphi_{n}(z) \in \mathcal{P}_{n-1}
$$

so

$$
\int\left(\psi_{n}(\zeta)-\frac{\gamma_{n}}{\kappa_{n}} \varphi_{n}(\zeta)\right) K_{n}(z, \zeta) d \mu(\zeta)=\psi_{n}(z)-\frac{\gamma_{n}}{\kappa_{n}} \varphi_{n}(z)
$$

by the reproducing property of $K_{n}$. Now using the orthogonality of $\varphi_{n}$, we can write

$$
\int\left(\psi_{n}(\zeta)-\frac{\gamma_{n}}{\kappa_{n}} \varphi_{n}(\zeta)\right) K_{n}(z, \zeta) d \mu(\zeta)=\int \psi_{n}(\zeta) K_{n}(z, \zeta) d \mu(\zeta)
$$

Further, on using the orthogonality of $\psi_{n}$ with respect to th inner product $\langle\cdot, \cdot\rangle$ defined above, we can rewrite the right side of the above equation as follows:

$$
\left\langle\psi_{n}, K_{n}(z, \cdot)\right\rangle-\psi_{n}^{\prime}(\boldsymbol{Z}) \boldsymbol{A} K_{n}^{(0,1)}(z, \boldsymbol{Z})^{t}=-\psi_{n}^{\prime}(\boldsymbol{Z}) \boldsymbol{A} K_{n}^{(0,1)}(z, \boldsymbol{Z})^{t} .
$$

This together with (4), establishes Formula 1.

Consequently, we have the following.

Formula 2. If $\psi_{n}$ exists, then

$$
\psi_{n}^{\prime}(z)-\frac{\gamma_{n}}{\kappa_{n}} \varphi_{n}^{\prime}(z)=-\psi_{n}^{\prime}(\boldsymbol{Z}) \boldsymbol{A} K_{n}^{(1,1)}(z, \boldsymbol{Z})^{t} .
$$

Proof. This formula follows from differentiating Formula 1 with respect to $z$ on both sides. 
Formula 3. If $\psi_{n}$ exists, then

$$
\frac{\gamma_{n}}{\kappa_{n}}=\left\langle\psi_{n}, \psi_{n}\right\rangle \frac{\kappa_{n}}{\gamma_{n}}-\psi_{n}^{\prime}(\boldsymbol{Z}) \boldsymbol{A} \varphi_{n}^{\prime}(\boldsymbol{Z})^{H}
$$

Proof. Consider different ways to calculate

$$
\int \psi_{n}(z) \overline{\varphi_{n}(z)} d \mu(z)
$$

Writing $\psi_{n}(z)=\left(\gamma_{n} / \kappa_{n}\right) \varphi_{n}(z)+$ lower degree terms, and using the orthogonality of $\varphi_{n}$, we have

$$
\int \psi_{n}(z) \overline{\varphi_{n}(z)} d \mu(z)=\frac{\gamma_{n}}{\kappa_{n}}
$$

On the other hand, writing $\varphi_{n}(z)=\left(\kappa_{n} / \gamma_{n}\right) \psi_{n}(z)+$ lower degree terms, and using the orthogonality of $\psi_{n}$, we have

$$
\begin{aligned}
\int \psi_{n}(z) \overline{\varphi_{n}(z)} d \mu(z) & =\left\langle\psi_{n}, \varphi_{n}\right\rangle-\psi_{n}^{\prime}(\boldsymbol{Z}) \boldsymbol{A} \varphi_{n}^{\prime}(\boldsymbol{Z})^{H} \\
& =\left\langle\psi_{n}, \psi_{n}\right\rangle \frac{\kappa_{n}}{\gamma_{n}}-\psi_{n}^{\prime}(\boldsymbol{Z}) \boldsymbol{A} \varphi_{n}^{\prime}(\boldsymbol{Z})^{H}
\end{aligned}
$$

which implies Formula 3.

Formula 4. Let $w_{m}(z):=\prod_{j=1}^{m}\left(1-\overline{z_{j}} z\right)$, then there exist two polynomials $p(z) \in \mathcal{P}_{2 m}$ and $q(z) \in \mathcal{P}_{2 m-1}$, uniquely determined by $\psi_{n}$, such that

$$
w_{m}^{2}(z) \psi_{n}(z)=\varphi_{n}(z) p(z)+\varphi_{n}^{*}(z) q(z) .
$$

Proof. From [6], we have

$$
\mathcal{P}_{n+2 m}=\varphi_{n} \mathcal{P}_{2 m}+\varphi_{n}^{*} \mathcal{P}_{2 m-1}+z^{2 m} \mathcal{P}_{n-2 m-1}
$$

So, there exist $p \in \mathcal{P}_{2 m}, q \in \mathcal{P}_{2 m-1}$ and $r \in \mathcal{P}_{n-2 m-1}$ such that

$$
w_{m}^{2}(z) \psi_{n}(z)=\varphi_{n}(z) p(z)+\varphi_{n}^{*}(z) q(z)+z^{2 m} r(z) .
$$

It remains to show $r \equiv 0$. Multiplying $\overline{z^{2 m} r(z)}$ and integrating with respect to $d \mu$ gives us

$$
\begin{aligned}
\int \psi_{n}(z) \overline{\left(w_{m}^{*}(z)\right)^{2} r(z)} d \mu= & \int \varphi_{n}(z) \overline{p^{*}(z) r(z)} d \mu+ \\
& \int \varphi_{n}^{*}(z) \overline{z q(z) r(z)} d \mu+\int|r(z)|^{2} d \mu
\end{aligned}
$$


where we have used the fact that $h_{k}^{*}(z)=z^{k} \overline{h(z)}$ for $|z|=1$. Now, by the orthogonality of $\varphi_{n}$ and $\psi_{n}$ it then follows that $\int|r(z)|^{2} d \mu=0$, which implies $r \equiv 0$.

Remark. 1. We emphasize that the two polynomials $p(z)$ and $q(z)$ in Formula 4 depend on $w_{m}(z)$ and $\psi_{n}(z)$, although the dependence is not given explicitly in the notation.

2. Formula 4 can be used to produce reccurence relation and determinantal representation for $\psi_{n}$. The following integral representation is just one of various other possibilities.

Formula 5. If $\left|z_{j}\right|>1, j=1,2, \ldots, m$, then

$$
q(z)=\frac{1}{2 \pi i} \int_{|\zeta|=1} \frac{\varphi_{n}(\zeta) p(\zeta)\left(w_{m}^{2}(z)-w_{m}^{2}(\zeta)\right)}{\varphi_{n}^{*}(\zeta) w_{m}^{2}(\zeta)(\zeta-z)} d \zeta,|z|<1
$$

and

$$
\psi_{n}(z)=\frac{\varphi_{n}^{*}(z)}{2 \pi i} \int_{|\zeta|=1} \frac{\varphi_{n}(\zeta) p(\zeta)}{\varphi_{n}^{*}(\zeta) w_{m}^{2}(\zeta)(\zeta-z)} d \zeta,|z|<1
$$

Proof. Using Formula 4, on substituting $z$ by $1 / \overline{z_{j}}$, we have

$$
q\left(\frac{1}{\overline{z_{j}}}\right)=-\left(\frac{\varphi_{n}}{\varphi_{n}^{*}} p\right)\left(\frac{1}{\overline{z_{j}}}\right), j-1,2, \ldots, m
$$

and

$$
q^{\prime}\left(\frac{1}{\overline{z_{j}}}\right)=-\left(\frac{\varphi_{n}}{\varphi_{n}^{*}} p\right)^{\prime}\left(\frac{1}{\overline{z_{j}}}\right), j-1,2, \ldots, m
$$

Now, Formula 5 follows from an application of the Hermite formula of interpolation (see, for example, [4, p. 68]).

\section{Relative Asymptotics.}

We now assume the matrix $\boldsymbol{A}$ in (3) is non-singular. Denote the leading coefficient of $\varphi_{n}$ by $\kappa_{n}>0$ as before. We need some assumptions on the measure $d \mu$. If

$$
\lim _{n \rightarrow \infty} \frac{\varphi_{n}(0)}{\kappa_{n}}=0,
$$

we say that the measure $d \mu$ belongs to class $N$ and write $d \mu \in N$. This is analogous to the Nevai's class of measures supported on the real line $\mathbb{R}$, (cf. 
[11]). A well known result of Rakhmanov (cf. [14]) says that any measure $d \mu$ with $\mu^{\prime}>0$ a.e. on $|z|=1$ belongs to class $N$.

We now state our main results on relative asymptotics, their proofs are presented in the next section.

Theorem 1. If the points $z_{1} \ldots, z_{m}\left(z_{j} \neq z_{k}\right.$ for $\left.j \neq k\right)$ all lie outside the unit circle, the matrix $\boldsymbol{A}$ in (3) is non-singular, and $d \mu \in N$, then there exists a positive integer $n_{0}$ such that for each $n \geq n_{0}$ the orthonormal polynomials with respect to the indefinite inner product $\langle\cdot, \cdot\rangle, \psi_{n}$, exists.

Recall that $\gamma_{n}>0$ denotes the leading coefficient of $\psi_{n}$ and $\left|\left\langle\psi_{n}, \psi_{n}\right\rangle\right|=1$.

Theorem 2. Under the same assumptions as in Theorem 1, there hold

$$
\lim _{n \rightarrow \infty} \frac{\gamma_{n}}{\kappa_{n}}=\frac{1}{\prod_{i=1}^{m}\left|z_{i}\right|}
$$

and

$$
\lim _{n \rightarrow \infty} \frac{\psi_{n}(z)}{\varphi_{n}(z)}=\lambda B(z),|z|>1
$$

where $\lambda=|B(0)| / B(0)$ and $B(z)=w_{m}^{*}(z) / w_{m}(z)$. (Recall that $w_{m}(z)=$ $\left.\prod_{j=1}^{m}\left(1-\overline{z_{j}} z\right).\right)$ The convergence in (8) is uniform on every compact subset in $|z|>1$.

Corollary 3. Let $\rho:=\min \left\{\left|z_{j}\right| \mid j=1,2, \ldots, m\right\}$. Then under the same assumption as in Theorem 1 we have

$$
\lim _{n \rightarrow \infty} \rho^{\epsilon n} \psi_{n}^{\prime}\left(z_{j}\right)=0
$$

for every $\epsilon \in(0,1)$ and $j=1,2, \ldots, m$.

Corollary 4. Let $p(z)$ be defined as in Formula 4. Under the same assumption as in Theorem 1, we have

$$
\lim _{n \rightarrow \infty} p(z)=\lambda w_{m}(z) w_{m}^{*}(z)=\lambda w_{m}^{2}(z) B(z)
$$

uniformly on every compact subset in the complex plane $\mathbb{C}$.

\section{Proofs of the Relative Asymptotics.}

We first establish some auxiliary lemmas. 
Lemma 5. For points $z_{1}, \ldots, z_{m}\left(z_{j} \neq z_{k}\right.$ for $\left.j \neq k\right)$ outside the unit circle, the matrix

$$
\boldsymbol{T}_{m}:=\left(\frac{1}{\overline{z_{j}} z_{k}-1}\right)_{j, k=1}^{m}
$$

is non-singular.

Proof. This result is known and follows from Cauchy's result that

$$
\operatorname{det}\left(\frac{1}{a_{i}+b_{j}}\right)_{i, j=1,2, \ldots, n}=\frac{\prod_{j>k}\left(a_{j}-a_{k}\right)\left(b_{j}-b_{k}\right)}{\prod_{j, k=1}^{n}\left(a_{j}+a_{k}\right)}
$$

(see, for example, [13, Problem 3 p. 92]. See also [7, Lemma 4].

Lemma 6. For points $z_{1}, \ldots, z_{m}\left(z_{j} \neq z_{k}\right.$ for $\left.j \neq k\right)$ outside the unit circle, let $B(z)=w_{m}^{*}(z) / w_{m}(z)$, then there exists a unique set of non-zero complex numbers $r_{1}, \ldots, r_{m}$ such that

$$
B(z)=\frac{1}{\overline{B(0)}}+\sum_{j=1}^{m} \frac{r_{j}}{1-\overline{z_{j}} z} .
$$

Proof. See [7, Lemma 5].

Lemma 7. If $d \mu$ is a positive finite measure on the unit circle with infinitely many points in the support, then for every compact subset $K$ in $|z|>1$ there exists two positive constants $d=d(K)$ and $e=e(K)$ independent of $n$ such that

$$
d \leq\left|\frac{\varphi_{n}^{\prime}(z)}{n \varphi_{n}(z)}\right| \leq e
$$

for all $z \in K$.

Proof. Denote $c:=\min \{|z| ; z \in K\}$ and $C:=\max \{|z| ; z \in K\}$. Then $1<$ $c<C$. To obtain the lower bound, write

$$
\frac{\varphi_{n}^{\prime}(z)}{n \varphi_{n}(z)}=\frac{1}{n} \sum_{j=1}^{n}\left\{\frac{\bar{z}}{\left|z-\zeta_{j}\right|^{2}}-\frac{\overline{\zeta_{j}}}{\left|z-\zeta_{j}\right|^{2}}\right\},
$$

where $\zeta_{j}$ 's denote the zeroes of $\varphi_{n}$ which are all in $|z|<1$, cf. [15, Th. 11.4.1.]. Then

$$
\begin{aligned}
\left|\frac{\varphi_{n}^{\prime}(z)}{n \varphi_{n}(z)}\right| & \geq \frac{|z|}{n} \sum_{j=1}^{n} \frac{1}{\left|z-\zeta_{j}\right|^{2}}-\frac{1}{n} \sum_{j=1}^{n} \frac{\left|\zeta_{j}\right|}{\left|z-\zeta_{j}\right|^{2}} \\
& =\frac{1}{n} \sum_{j=1}^{n} \frac{|z|-\left|\zeta_{j}\right|}{\left|z-\zeta_{j}\right|^{2}} \geq \frac{c-1}{(C+1)^{2}} .
\end{aligned}
$$


The proof of the upper bound is easier.

Remark. Lemma 7 and its proof are parallel to those of Lemma 3 in [5].

Lemma 8. If $d \mu \in N$, then

$$
\lim _{n \rightarrow \infty} \frac{\varphi_{n+1}^{\prime}(z)}{\varphi_{n}^{\prime}(z)}=z
$$

uniformly on every compact subset of $|z|>1$. Furthermore,

$$
\lim _{n \rightarrow \infty} \frac{K_{n}^{(0,1)}(z, \zeta)}{\varphi_{n}^{\prime}(z) \overline{\varphi_{n}(\zeta)}}=\frac{1}{z \bar{\zeta}-1}
$$

and

$$
\lim _{n \rightarrow \infty} \frac{K_{n}^{(1,1)}(z, \zeta)}{\varphi_{n}^{\prime}(z) \overline{\varphi_{n}^{\prime}(\zeta)}}=\frac{1}{z \bar{\zeta}-1}
$$

uniformly for $(z, \zeta)$ on every compact subset of $|z|>1$ and $|\zeta|>1$.

Proof. If $d \mu \in N$, then by $[\mathbf{1 2}, \mathbf{1 4}]$

$$
\lim _{n \rightarrow \infty} \frac{\varphi_{n+1}(z)}{\varphi_{n}(z)}=z
$$

uniformly on every compact subset of $|z|>1$. As in [5, Lemma 4], note that

$$
\frac{\varphi_{n+1}^{\prime}(z)}{\varphi_{n}^{\prime}(z)}=\frac{\varphi_{n}(z)}{\varphi_{n}^{\prime}(z)}\left(\frac{\varphi_{n+1}}{\varphi_{n}}\right)^{\prime}(z)+\frac{\varphi_{n+1}(z)}{\varphi_{n}(z)} .
$$

Now (12) implies

$$
\lim _{n \rightarrow \infty}\left(\frac{\varphi_{n+1}}{\varphi_{n}}\right)^{\prime}(z)=1,
$$

uniformly on every compact subset of $|z|>1$. On the other hand, by Lemma 7 ,

$$
\lim _{n \rightarrow \infty} \frac{\varphi_{n}(z)}{\varphi_{n}^{\prime}(z)}=0
$$

uniformly on every compact subset of $|z|>1$. Thus, on taking the limit as $n$ tends to $\infty$ on both sides of (13), we obtain

$$
\lim _{n \rightarrow \infty} \frac{\varphi_{n+1}^{\prime}(z)}{\varphi_{n}^{\prime}(z)}=z
$$


uniformly on every compact subset of $|z|>1$. This proves (9).

In order to prove (11), we need the following limit relations:

$$
\lim _{n \rightarrow \infty} \frac{\varphi_{n}^{*}(z)}{\varphi_{n}(z)}=0
$$

and

$$
\lim _{n \rightarrow \infty} \frac{\varphi_{n}^{* \prime}(z)}{\varphi_{n}^{\prime}(z)}=0,
$$

uniformly on every compact subset of $|z|>1$. Relation (15) is established in the proof of Theorem 4 in [12], while relation (16) folows from (14), (15) and the identity

$$
\frac{\varphi_{n}^{* \prime}(z)}{\varphi_{n}^{\prime}(z)}=\frac{\varphi_{n}(z)}{\varphi_{n}^{\prime}(z)}\left(\frac{\varphi_{n}^{*}}{\varphi_{n}}\right)^{\prime}(z)+\frac{\varphi_{n}^{*}(z)}{\varphi_{n}(z)} .
$$

Now, using the Christoffel-Darboux formula (cf. [15, Th. 11.4.2.]) and by straightforward calculations, we have

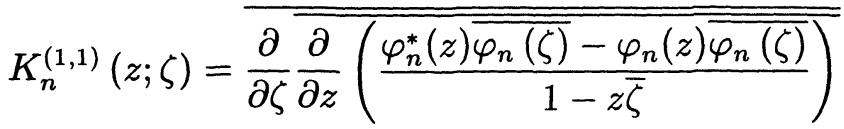

$$
\begin{aligned}
& =\frac{\varphi_{n}^{* \prime}(z) \overline{\varphi_{n}^{* \prime}(\zeta)}-\varphi_{n}^{\prime}(z) \overline{\varphi_{n}^{\prime}(\zeta)}}{1-z \bar{\zeta}}+\frac{\varphi_{n}^{* \prime}(z) \overline{\varphi_{n}^{*}(\zeta)}-\varphi_{n}^{\prime}(z) \overline{\varphi_{n}(\zeta)}}{(1-z \bar{\zeta})^{2}} z \\
& \frac{\varphi_{n}^{*}(z) \overline{\varphi_{n}^{* \prime}(\zeta)}-\varphi_{n}(z) \overline{\varphi_{n}^{\prime}(\zeta)}}{(1-z \bar{\zeta})^{2}} \bar{\zeta}+\frac{\varphi_{n}^{*}(z) \overline{\varphi_{n}^{*}(\zeta)}-\varphi_{n}(z) \overline{\varphi_{n}(\zeta)}}{(1-z \bar{\zeta})^{3}}(1+\bar{z} \zeta) \\
& =: I_{1}+I_{2}+I_{3}+I_{4} \text {. }
\end{aligned}
$$

It is easy to verify that

$$
\frac{I_{1}}{\varphi_{n}^{\prime}(z) \overline{\varphi_{n}^{\prime}(\zeta)}}=\frac{1}{1-z \bar{\zeta}}\left(\frac{\varphi_{n}^{* \prime}(z)}{\varphi_{n}^{\prime}(z)} \overline{\left(\frac{\varphi_{n}^{* \prime}(\zeta)}{\varphi_{n}^{\prime}(\zeta)}\right)}-1\right) \rightarrow \frac{1}{z \bar{\zeta}-1},
$$

and

$$
\frac{I_{4}}{\varphi_{n}^{\prime}(z) \overline{\varphi_{n}^{\prime}(\zeta)}}=\frac{1+\bar{z} \zeta}{(1-z \bar{\zeta})^{3}}\left(\frac{\varphi_{n}^{*}(z)}{\varphi_{n}(z)} \overline{\left(\frac{\varphi_{n}^{*}(\zeta)}{\varphi_{n}(\zeta)}\right)}-1\right) \frac{\varphi_{n}(z)}{\varphi_{n}^{\prime}(z)} \overline{\left(\frac{\varphi_{n}(\zeta)}{\varphi_{n}^{\prime}(\zeta)}\right)} \rightarrow 0
$$

as $n \rightarrow \infty$, by (14), (15) and (16). Also, on writing

$$
\frac{I_{2}}{\varphi_{n}^{\prime}(z) \overline{\varphi_{n}^{\prime}(\zeta)}}=\frac{z}{(1-z \bar{\zeta})^{2}}\left(\frac{\varphi_{n}^{* \prime}(z)}{\varphi_{n}^{\prime}(z)} \overline{\left(\frac{\varphi_{n}^{*}(\zeta) \varphi_{n}(\zeta)}{\varphi_{n}(\zeta) \varphi_{n}^{\prime}(\zeta)}\right)}-\overline{\left(\frac{\varphi_{n}(\zeta)}{\varphi_{n}^{\prime}(\zeta)}\right)}\right)
$$


we get, by (14), (15) and (16)

$$
\frac{I_{2}}{\varphi_{n}^{\prime}(z) \overline{\varphi_{n}^{\prime}(\zeta)}} \rightarrow 0
$$

as $n \rightarrow \infty$. Similarly, we can obtain

$$
\frac{I_{3}}{\varphi_{n}^{\prime}(z) \overline{\varphi_{n}^{\prime}(\zeta)}} \rightarrow 0
$$

as $n \rightarrow \infty$.

Note that all above limit processes are uniform for compact subsets in $|z|>1$ and $|\zeta|>1$. So (11) holds.

The proof of (10) is similar to that of (11), so we omit it.

Proof of Theorem 1. We first claim that there exists a positive integer $n_{0}$ such that

$$
\operatorname{det}\left(\boldsymbol{A}^{-1}+\boldsymbol{K}_{n}^{(1,1)}\right) \neq 0, n \geq n_{0}
$$

where

$$
\boldsymbol{K}_{n}^{(1,1)}:=\left(\begin{array}{cccc}
K_{n}^{(1,1)}\left(z_{1}, z_{1}\right) & K_{n}^{(1,1)}\left(z_{2}, z_{1}\right) & \ldots & K_{n}^{(1,1)}\left(z_{m}, z_{1}\right) \\
K_{n}^{(1,1)}\left(z_{1}, z_{2}\right) & K_{n}^{(1,1)}\left(z_{2}, z_{2}\right) & \ldots & K_{n}^{(1,1)}\left(z_{m}, z_{2}\right) \\
\ldots & \ldots & \ldots \\
K_{n}^{(1,1)}\left(z_{1}, z_{m}\right) & K_{n}^{(1,1)}\left(z_{2}, z_{m}\right) & \ldots & K_{n}^{(1,1)}\left(z_{m}, z_{m}\right)
\end{array}\right)
$$

Assume the validity of this claim for the moment. Define

$$
\phi_{n}(z):=\varphi_{n}(z)-\varphi_{n}^{\prime}(\boldsymbol{Z})\left(\boldsymbol{I}+\boldsymbol{A} \boldsymbol{K}_{n}^{(1,1)}\right)^{-1} \boldsymbol{A} K_{n}^{(0,1)}(z, \boldsymbol{Z})^{t}, n \geq n_{0}
$$

It then follows that

$$
\phi_{n}^{\prime}(\boldsymbol{Z})=\varphi_{n}^{\prime}(\boldsymbol{Z})\left(\boldsymbol{I}+\boldsymbol{A} \boldsymbol{K}_{n}^{(1,1)}\right)^{-1} .
$$

Thus, for $k<n$,

$$
\begin{aligned}
& \left\langle\phi_{n}, \varphi_{k}\right\rangle=\int \phi_{n}(z) \overline{\varphi_{k}(z)} d \mu+\phi_{n}^{\prime}(\boldsymbol{Z}) \boldsymbol{A} \varphi_{k}^{\prime}(\boldsymbol{Z})^{H} \\
= & \int \varphi_{n}(z) \overline{\varphi_{k}(z)} d \mu-\varphi_{n}^{\prime}(\boldsymbol{Z})\left(\boldsymbol{I}+\boldsymbol{A} \boldsymbol{K}_{n}^{(1,1)}\right)^{-1} \boldsymbol{A} \int K_{n}^{(0,1)}(z, \boldsymbol{Z})^{t} \overline{\varphi_{k}(z)} d \mu \\
& \quad+\varphi_{n}^{\prime}(\boldsymbol{Z})\left(\boldsymbol{I}+\boldsymbol{A} \boldsymbol{K}_{n}^{(1,1)}\right)^{-1} \boldsymbol{A} \varphi_{k}^{\prime}(\boldsymbol{Z})^{H} \\
= & 0 .
\end{aligned}
$$


Similarly,

$$
\begin{aligned}
& \left\langle\phi_{n}, \varphi_{n}\right\rangle=\int \phi_{n}(z) \overline{\varphi_{n}(z)} d \mu+\phi_{n}^{\prime}(\boldsymbol{Z}) \boldsymbol{A} \varphi_{n}^{\prime}(\boldsymbol{Z})^{H} \\
= & 1+\varphi_{n}^{\prime}(\boldsymbol{Z})\left(\boldsymbol{I}+\boldsymbol{A} \boldsymbol{K}_{n}^{(1,1)}\right)^{-1} \boldsymbol{A} \varphi_{n}^{\prime}(\boldsymbol{Z})^{H} .
\end{aligned}
$$

Now, from the matrix identities

$$
\begin{aligned}
& \left(\begin{array}{cc}
\boldsymbol{I}_{m \times m} \varphi_{n}^{\prime}(\boldsymbol{Z})^{H} \\
\mathbf{0}_{1 \times m} & 1
\end{array}\right)\left(\begin{array}{cc}
\boldsymbol{A}^{-1}+\boldsymbol{K}_{n}^{(1,1)} & -\varphi_{n}^{\prime}(\boldsymbol{Z})^{H} \\
\varphi_{n}^{\prime}(\boldsymbol{Z})^{-} & 1
\end{array}\right) \\
& =\left(\begin{array}{cc}
\boldsymbol{A}^{-1}+\boldsymbol{K}_{n}^{(1,1)}+\varphi_{n}^{\prime}(\boldsymbol{Z})^{H} & \varphi_{n}^{\prime}(\boldsymbol{Z}) \\
\boldsymbol{0}_{1 \times m} \\
\varphi_{n}^{\prime}(\boldsymbol{Z}) & 1
\end{array}\right)
\end{aligned}
$$

and

$$
\begin{aligned}
& \left(\begin{array}{cc}
\boldsymbol{A}^{-1}+\boldsymbol{K}_{n}^{(1,1)}-\varphi_{n}^{\prime}(\boldsymbol{Z})^{H} \\
\varphi_{n}^{\prime}(\boldsymbol{Z}) & 1
\end{array}\right)\left(\begin{array}{c}
\boldsymbol{I}_{m \times m}\left(\boldsymbol{A}^{-1}+\boldsymbol{K}_{n}^{(1,1)}\right)^{-1} \varphi_{n}^{\prime}(\boldsymbol{Z})^{H} \\
\mathbf{0}_{1 \times m}
\end{array}\right) \\
= & \left(\begin{array}{cc}
\boldsymbol{A}^{-1}+\boldsymbol{K}_{n}^{(1,1)} & \mathbf{0}_{m \times 1} \\
\varphi_{n}^{\prime}(\boldsymbol{Z}) & 1+\varphi_{n}^{\prime}(\boldsymbol{Z})\left(\boldsymbol{A}^{-1}+\boldsymbol{K}_{n}^{(1,1)}\right)^{-1} \varphi_{n}^{\prime}(\boldsymbol{Z})^{H}
\end{array}\right),
\end{aligned}
$$

we get the following determinant identity

$$
\begin{aligned}
& \operatorname{det}\left(\boldsymbol{A}^{-1}+\boldsymbol{K}_{n}^{(1,1)}+\varphi_{n}^{\prime}(\boldsymbol{Z})^{H} \varphi_{n}^{\prime}(\boldsymbol{Z})\right)= \\
& \operatorname{det}\left(\boldsymbol{A}^{-1}+\boldsymbol{K}_{n}^{(1,1)}\right)\left(1+\varphi_{n}^{\prime}(\boldsymbol{Z})\left(\boldsymbol{A}^{-1}+\boldsymbol{K}_{n}^{(1,1)}\right)^{-1} \varphi_{n}^{\prime}(\boldsymbol{Z})^{H}\right) .
\end{aligned}
$$

Note also that

$$
\boldsymbol{K}_{n+1}^{(1,1)}=\boldsymbol{K}_{n}^{(1,1)}+\varphi_{n}^{\prime}(\boldsymbol{Z})^{H} \varphi_{n}^{\prime}(\boldsymbol{Z})
$$

so there holds

$$
1+\varphi_{n}^{\prime}(\boldsymbol{Z})\left(\boldsymbol{A}^{-1}+\boldsymbol{K}_{n}^{(1,1)}\right)^{-1} \varphi_{n}^{\prime}(\boldsymbol{Z})^{H}=\frac{\operatorname{det}\left(\boldsymbol{A}^{-1}+\boldsymbol{K}_{n+1}^{(1,1)}\right)}{\operatorname{det}\left(\boldsymbol{A}^{-1}+\boldsymbol{K}_{n}^{(1,1)}\right)} .
$$

This together with (18), gives us

$$
\left\langle\phi_{n}, \varphi_{n}\right\rangle=\frac{\operatorname{det}\left(\boldsymbol{A}^{-1}+\boldsymbol{K}_{n+1}^{(1,1)}\right)}{\operatorname{det}\left(\boldsymbol{A}^{-1}+\boldsymbol{K}_{n}^{(1,1)}\right)} \neq 0, n \geq n_{0} .
$$

Therefore, a (left-) orthonormal polynomial $\psi_{n}$ exists and equals

$$
\psi_{n}(z)=\eta_{n} \frac{\phi_{n}(z)}{\left\langle\phi_{n}, \phi_{n}\right\rangle}
$$


where $\eta_{n}$ is a number of modulus 1 such that the above polynomial has a positive leading coefficient.

It remains to prove our claim (17). Assume, to the contrary, that there is an infinite subsequence of positive integers, say $\mathcal{L}$, such that

$$
\operatorname{det}\left(\boldsymbol{A}^{-1}+\boldsymbol{K}_{n}^{(1,1)}\right)=0, \text { for all } n \in \mathcal{L} .
$$

Let

$$
\Lambda_{n}:=\left(\begin{array}{cccc}
\frac{1}{\varphi_{n}^{\prime}\left(z_{1}\right)} & 0 & \ldots & 0 \\
0 & \frac{1}{\varphi_{n}^{\prime}\left(z_{2}\right)} & \ldots & 0 \\
\ldots & \ldots & & \ldots \\
0 & 0 & \ldots & \frac{1}{\varphi_{n}^{\prime}\left(z_{m}\right)}
\end{array}\right)
$$

and

$$
\boldsymbol{T}_{m, n}:=\left(\begin{array}{cccc}
\frac{K_{n}^{(1,1)}\left(z_{1}, z_{1}\right)}{\varphi_{n}^{\prime}\left(z_{1}\right) \overline{\varphi_{n}^{\prime}\left(z_{1}\right)}} & \frac{K_{n}^{(1,1)}\left(z_{2}, z_{1}\right)}{\varphi_{n}^{\prime}\left(z_{2}\right) \bar{\varphi}_{n}^{\prime}\left(z_{1}\right)} \cdots & \frac{K_{n}^{(1,1)}\left(z_{m}, z_{1}\right)}{\varphi_{n}^{\prime}\left(z_{m}\right) \overline{\varphi_{n}^{\prime}\left(z_{1}\right)}} \\
\frac{K_{n}^{(1,1)}\left(z_{1}, z_{2}\right)}{\varphi_{n}^{\prime}\left(z_{1}\right) \overline{\varphi_{n}^{\prime}\left(z_{2}\right)}} & \frac{K_{n}^{(1,1)}\left(z_{2}, z_{2}\right)}{\varphi_{n}^{\prime}\left(z_{2}\right) \bar{\varphi}_{n}^{\prime}\left(z_{2}\right)} \cdots & \frac{K_{n}^{(1), 1)}\left(z_{m}, z_{2}\right)}{\varphi_{n}^{\prime}\left(z_{m}\right) \bar{\varphi}_{n}^{\prime}\left(z_{2}\right)} \\
\ldots & \ldots & \ldots & \ldots \\
\frac{K_{n}^{(1,1)}\left(z_{1}, z_{m}\right)}{\varphi_{n}^{\prime}\left(z_{1}\right) \bar{\varphi}_{n}^{\prime}\left(z_{m}\right)} & \frac{K_{n}^{(1,1)}\left(z_{2}, z_{m}\right)}{\varphi_{n}^{\prime}\left(z_{2}\right) \overline{\varphi_{n}^{\prime}\left(z_{m}\right)}} \cdots & \frac{K_{n}^{(1,1)}\left(z_{m}, z_{m}\right)}{\varphi_{n}^{\prime}\left(z_{m}\right) \varphi_{n}^{\prime}\left(z_{m}\right)}
\end{array}\right) .
$$

Then we can write

$$
A^{-1}+K_{n}^{(1,1)}=\overline{\Lambda_{n}^{-1}}\left(\overline{\Lambda_{n}} A^{-1} \Lambda_{n}+T_{m, n}\right) \Lambda_{n}^{-1} .
$$

Taking the determinant on both sides yields

$$
\operatorname{det}\left(\overline{\Lambda_{n}} A^{-1} \Lambda_{n}+T_{m, n}\right)=0, \text { for all } n \in \mathcal{L},
$$

according to (19). Now, using (9) in Lemma 8, we have

$$
\lim _{n \rightarrow \infty} \frac{1}{\varphi_{n}^{\prime}\left(z_{j}\right)}=0, j=1,2, \ldots, m
$$

and thus

$$
\lim _{n \rightarrow \infty} \Lambda_{n}=0
$$

while using (11), we get

$$
\lim _{n \rightarrow \infty} T_{m, n}=T_{m}
$$

Thus, if we let $n \in \mathcal{L}$ and $n \rightarrow \infty$ in (20), we would have $\operatorname{det} \boldsymbol{T}_{\boldsymbol{m}}=0$, contradicting Lemma 5 . So, the claim (17) must be true. 
Proof of Theorem 2. Let $n_{0}$ be defined as in Theorem 1. Assume $n \geq n_{0}$ in this proof. Our proof exploits the information obtained in the proof of Theorem 1. Let $z=z_{j}, j=1,2, \ldots, m$, in Formula 2,

$$
\psi_{n}^{\prime}\left(z_{j}\right)-\frac{\gamma_{n}}{\kappa_{n}} \varphi_{n}^{\prime}\left(z_{j}\right)=-\psi_{n}^{\prime}(\boldsymbol{Z}) \boldsymbol{A} K_{n}^{(1,1)}\left(z_{j}, \boldsymbol{Z}\right)^{t}, j=1,2, \ldots, m .
$$

Rearranging the terms in the above equations, and then putting them into a matrix form, we get

$$
\frac{\gamma_{n}}{\kappa_{n}}(1,1, \ldots, 1)=\psi_{n}^{\prime}(\boldsymbol{Z}) \boldsymbol{A} \boldsymbol{K}_{n}^{(1,1)} \boldsymbol{\Lambda}_{n}+\psi_{n}^{\prime}(\boldsymbol{Z}) \boldsymbol{\Lambda}_{\boldsymbol{n}}
$$

where $\boldsymbol{\Lambda}_{\boldsymbol{n}}$ is the same as in the proof of Theorem 1, Rewrite the above equation further as

$$
(1,1, \ldots, 1)=\frac{\kappa_{n}}{\gamma_{n}} \psi_{n}^{\prime}(\boldsymbol{Z})\left[\boldsymbol{A} \overline{\mathbf{\Lambda}_{n}^{-1}} \boldsymbol{T}_{\boldsymbol{m}, \boldsymbol{n}}+\boldsymbol{\Lambda}_{\boldsymbol{n}}\right],
$$

where $\boldsymbol{T}_{\boldsymbol{m}, \boldsymbol{n}}$ is as in the proof of Theorem 1. Using (20), (21) and (22), we obtain

$$
\begin{aligned}
& \lim _{n \rightarrow \infty} \frac{\kappa_{n}}{\gamma_{n}} \psi_{n}^{\prime}(\boldsymbol{Z}) \overline{\boldsymbol{A}} \overline{\boldsymbol{\Lambda}_{n}^{-1}} \\
= & \lim _{n \rightarrow \infty} \frac{\kappa_{n}}{\gamma_{n}} \psi_{n}^{\prime}(\boldsymbol{Z}) \boldsymbol{A} \overline{\boldsymbol{\Lambda}_{n}^{-1}}\left[\boldsymbol{T}_{\boldsymbol{m}, \boldsymbol{n}}+\overline{\boldsymbol{\Lambda}_{n}} \boldsymbol{A}^{-1} \boldsymbol{\Lambda}_{\boldsymbol{n}}\right]\left[\boldsymbol{T}_{\boldsymbol{m}, \boldsymbol{n}}+\overline{\boldsymbol{\Lambda}_{\boldsymbol{n}}} \boldsymbol{A}^{-1} \boldsymbol{\Lambda}_{\boldsymbol{n}}\right]^{-1} \\
= & \lim _{n \rightarrow \infty}(1,1, \ldots, 1)\left[\boldsymbol{T}_{\boldsymbol{m}, \boldsymbol{n}}+\overline{\boldsymbol{\Lambda}_{\boldsymbol{n}}} \boldsymbol{A}^{-1} \boldsymbol{\Lambda}_{\boldsymbol{n}}\right]^{-1} \\
= & (1,1, \ldots, 1) \boldsymbol{T}_{\boldsymbol{m}}^{-1}=\overline{B(0)}\left(r_{1}, r_{2}, \ldots, r_{m}\right),
\end{aligned}
$$

where the last equality is according to Lemma 6 when $z=0$.

Write $\boldsymbol{A}=\left(\boldsymbol{a}_{\mathbf{1}}^{\boldsymbol{t}}, \boldsymbol{a}_{\mathbf{2}}^{\boldsymbol{t}}, \ldots, \boldsymbol{a}_{\boldsymbol{m}}^{\boldsymbol{t}}\right)$ with $\boldsymbol{a}_{\boldsymbol{j}}=\left(a_{1 j}, a_{2 j}, \ldots, a_{m j}\right)(j=1,2, \ldots, m)$, then we have

$$
\lim _{n \rightarrow \infty} \frac{\kappa_{n}}{\gamma_{n}} \psi_{n}^{\prime}(\boldsymbol{Z}) \boldsymbol{a}_{j}^{\boldsymbol{t}} \overline{\varphi_{n}\left(z_{j}\right)}=\overline{B(0)} r_{j},, j=1,2, \ldots, m .
$$

Now, by Formula 3,

$$
\left\langle\psi_{n}, \psi_{n}\right\rangle\left(\frac{\kappa_{n}}{\gamma_{n}}\right)^{2}-1=\frac{\kappa_{n}}{\gamma_{n}} \psi_{n}^{\prime}(\boldsymbol{Z})\left(\boldsymbol{a}_{\mathbf{1}}^{\boldsymbol{t}}, \boldsymbol{a}_{\mathbf{2}}^{t}, \ldots, \boldsymbol{a}_{m}^{\boldsymbol{t}}\right) \varphi_{n}^{\prime}(\boldsymbol{Z})^{H},
$$

thus

$$
\begin{aligned}
\lim _{n \rightarrow \infty}\left\langle\psi_{n}, \psi_{n}\right\rangle\left(\frac{\kappa_{n}}{\gamma_{n}}\right)^{2} & =1+\lim _{n \rightarrow \infty} \sum_{j=1}^{m} \frac{\kappa_{n}}{\gamma_{n}} \psi_{n}^{\prime}(\boldsymbol{Z}) \boldsymbol{a}_{j}^{t} \overline{\varphi_{n}^{\prime}\left(z_{j}\right)} \\
& =1+\overline{B(0)} \sum_{j=1}^{m} r_{j}=1+\overline{B(0)}\left[B(0)-\frac{1}{\overline{B(0)}}\right] \\
& =|B(0)|^{2} .
\end{aligned}
$$


Therefore $\lim _{n \rightarrow \infty}\left\langle\psi_{n}, \psi_{n}\right\rangle=1$ and

$$
\lim _{n \rightarrow \infty} \frac{\kappa_{n}}{\gamma_{n}}=|B(0)|=\frac{1}{\prod_{j=1}^{m}\left|z_{j}\right|} .
$$

This completes the proof of (7).

We now prove (8). From Formula 1,

$$
\frac{\psi_{n}(z)}{\varphi_{n}(z)}=\frac{\gamma_{n}}{\kappa_{n}}-\psi_{n}^{\prime}(\boldsymbol{Z}) \boldsymbol{A} \frac{K_{n}^{(0.1)}(z, \boldsymbol{Z})^{t}}{\varphi_{n}(z)}
$$

Recall that $K_{n}^{(0,1)}(z, \boldsymbol{Z})=\left(K_{n}^{(0,1)}\left(z, z_{1}\right), \ldots, K_{n}^{(0,1)}\left(z, z_{m}\right)\right)$. Using $\boldsymbol{A}=$ $\left(\boldsymbol{a}_{1}^{t}, \ldots, \boldsymbol{a}_{m}^{t}\right)$ and expanding the product on the right-hand side in the above equation, we have

$$
\frac{\psi_{n}(z)}{\varphi_{n}(z)}=\frac{\gamma_{n}}{\kappa_{n}}-\sum_{j=1}^{m} \psi_{n}^{\prime}(\boldsymbol{Z}) \boldsymbol{a}_{j}^{\boldsymbol{t}} \overline{\varphi_{n}^{\prime}\left(z_{j}\right)} \frac{K_{n}^{(0,1)}\left(z, z_{j}\right)}{\varphi_{n}(z) \overline{\varphi_{n}^{\prime}\left(z_{j}\right)}} .
$$

Letting $n \rightarrow \infty$ on both sides in the above equation yields

$$
\lim _{n \rightarrow \infty} \frac{\psi_{n}(z)}{\varphi_{n}(z)}=\frac{1}{|B(0)|}-\sum_{j=1}^{m} \overline{B(0)} r_{j} \frac{1}{z \overline{z_{j}}-1}
$$

by (7), (23) and (10). It follows from Lemma 6 and the definition of $\lambda$ that the right-hand side in the above equation is $\lambda B(z)$. So (8) holds, and the proof of the theorem is completed.

Proof of Corollary 3. From (23) and (7),

$$
\lim _{n \rightarrow \infty} \psi_{n}^{\prime}(\boldsymbol{Z}) \boldsymbol{a}_{j}^{t} \overline{\varphi_{n}\left(z_{j}\right)}=\frac{\overline{B(0)}}{|B(0)|} \boldsymbol{r}
$$

where $r:=\left(r_{1}, r_{2}, \ldots, r_{m}\right)$. So

$$
\psi_{n}^{\prime}(\boldsymbol{Z}) \overline{\boldsymbol{A} \boldsymbol{\Lambda}_{n}^{-1}}=\frac{\overline{B(0)}}{|B(0)|} \boldsymbol{r}+\boldsymbol{f}_{m}
$$

where $\lim _{n \rightarrow \infty} f_{m}=\mathbf{0}_{m}$. Thus

$$
\psi_{n}^{\prime}(\boldsymbol{Z})=\frac{\overline{B(0)}}{|B(0)|} \boldsymbol{r} \overline{\boldsymbol{\Lambda}_{n}} \boldsymbol{A}^{-1}+\boldsymbol{f}_{m} \overline{\boldsymbol{\Lambda}_{n}} \boldsymbol{A}^{-1},
$$

and so $\lim _{n \rightarrow \infty} \rho^{\epsilon n} \psi_{n}^{\prime}(\boldsymbol{Z})=\mathbf{0}_{m}$, according to (9). 
Proof of Corollary 4. Denote

$$
\alpha_{n, j}:=p\left(\frac{1}{\overline{z_{j}}}\right), \text { and } \beta_{n, j}:=p^{\prime}\left(\frac{1}{\overline{z_{j}}}\right),
$$

for $j=1,2, \ldots, m$. Choose $\lambda_{n}$ such that $p(z)-\lambda_{n} w_{m}^{2}(z) \in \mathcal{P}_{2 m-1}$. Since $\operatorname{deg} \psi_{n}=n$ for $n \geq n_{0}$, by comparing the leading coefficients in Formula 4, we find that $\lambda_{n}=\frac{\gamma_{n}}{\kappa_{n}}$.

Recall the formula of the $(0,1)$ polynomial Hermite interpolation (cf. $[9$, p. 300]): for a differentiable function $f$,

$$
H_{2 m-1}(z, f)=\sum_{j=1}^{m} f\left(\frac{1}{\overline{z_{j}}}\right) s_{j}(z)+\sum_{j=1}^{m} f^{\prime}\left(\frac{1}{\overline{z_{j}}}\right) t_{j}(z)
$$

is the interpolating polynomial of the degree at most $2 m-1$ satisfying

$$
H_{2 m-1}\left(\frac{1}{\overline{z_{j}}}\right)=f\left(\frac{1}{\overline{z_{j}}}\right), H_{2 m-1}^{\prime}\left(\frac{1}{\overline{z_{j}}}\right)=f^{\prime}\left(\frac{1}{\overline{z_{j}}}\right),
$$

for $j=1,2, \ldots, m$, where the polynomials $s_{j}(z)$ and $t_{j}(z)$ are given by

$$
\begin{gathered}
s_{j}(z)=v_{j}(z) L_{j}(z)^{2}, t_{j}(z)=\left(z-\frac{1}{\overline{z_{j}}}\right) L_{j}(z)^{2}, \\
v_{j}(z)=1-\frac{w_{m}^{\prime \prime}\left(\frac{1}{\bar{z}_{j}}\right)}{w_{m}^{\prime}\left(\frac{1}{\overline{z_{j}}}\right)}\left(z-\frac{1}{\overline{z_{j}}}\right), \quad L_{j}(z)=\frac{w_{m}(z)}{\left(z-\frac{1}{\bar{z}_{j}}\right) w_{m}^{\prime}\left(\frac{1}{\bar{z}_{j}}\right)},
\end{gathered}
$$

for $j=1,2, \ldots, m$. When $f$ is a polynomial of degree $\leq 2 m-1$, formula (24) reproduces $f$. In particular, for $f(z)=p(z)-\lambda_{n} w_{m}^{2}(z)$,

$$
p(z)-\lambda_{n} w_{m}^{2}(z)=\sum_{j=1}^{m} \alpha_{n, j} s_{j}(z)+\sum_{j=1}^{m} \beta_{n, j} t_{j}(z) .
$$

On the other hand, using (5), (6) and (25), we see that

$$
\begin{aligned}
q(z)= & -\sum_{j=1}^{m} \frac{\varphi_{n}}{\varphi_{n}^{*}}\left(\frac{1}{\overline{z_{j}}}\right) \alpha_{n, j} s_{j}(z) \\
& -\sum_{j=1}^{m}\left\{\left(\frac{\varphi_{n}}{\varphi_{n}^{*}}\right)^{\prime}\left(\frac{1}{\overline{z_{j}}}\right) \alpha_{n, j}+\frac{\varphi_{n}}{\varphi_{n}^{*}}\left(\frac{1}{\overline{z_{j}}}\right) \beta_{n, j}\right\} t_{j}(z) .
\end{aligned}
$$

Substituting (25) and (26) into Formula 4 yields

$$
\begin{aligned}
& w_{m}^{2}(z) \psi_{n}(z)=\lambda_{n} w_{m}^{2}(z) \varphi_{n}(z)+\sum_{j=1}^{m}\left[\varphi_{n}(z)-\varphi_{n}^{*}(z) \frac{\varphi_{n}}{\varphi_{n}^{*}}\left(\frac{1}{\overline{z_{j}}}\right)\right] \alpha_{n, j} s_{\jmath}(z) \\
+ & \sum_{j=1}^{m}\left[\varphi_{n}(z)-\varphi_{n}^{*}(z) \frac{\varphi_{n}}{\varphi_{n}^{*}}\left(\frac{1}{\overline{z_{j}}}\right)\right] \beta_{n, j} t_{j}(z)-\sum_{j=1}^{m} \varphi_{n}^{*}(z)\left(\frac{\varphi_{n}}{\varphi_{n}^{*}}\right)^{\prime}\left(\frac{1}{\overline{z_{j}}}\right) \alpha_{n, j} t_{\jmath}(z) .
\end{aligned}
$$


Note that, by the Christoffel-Darboux formula,

$$
\varphi_{n}(z)-\varphi_{n}^{*}(z) \frac{\varphi_{n}}{\varphi_{n}^{*}}\left(\frac{1}{\overline{z_{j}}}\right)=\frac{K_{n}\left(z, z_{j}\right)\left(\overline{z_{j}} z-1\right)}{\overline{\varphi_{n}\left(z_{j}\right)}} .
$$

So

$$
\begin{gathered}
\frac{w_{m}^{2} \psi_{n}}{\varphi_{n}}(z)=\lambda_{n} w_{m}^{2}(z)+\sum_{j=1}^{m} \frac{K_{n}\left(z, z_{j}\right)\left(\overline{z_{j}} z-1\right)}{\varphi_{n}(z) \overline{\varphi_{n}\left(z_{j}\right)}} \alpha_{n, j} s_{j}(z) \\
+\sum_{j=1}^{m} \frac{K_{n}\left(z, z_{j}\right)\left(\overline{z_{j}} z-1\right)}{\varphi_{n}(z) \overline{\varphi_{n}\left(z_{j}\right)}} \beta_{n, j} t_{j}(z)-\sum_{j=1}^{m} \frac{\varphi_{n}^{*}}{\varphi_{n}}(z)\left(\frac{\varphi_{n}}{\varphi_{n}^{*}}\right)^{\prime}\left(\frac{1}{\overline{z_{j}}}\right) \alpha_{n, j} t_{j}(z) .
\end{gathered}
$$

Taking arbitrary $2 m$ distinct points in $|z|>1$, say $\zeta_{1}, \zeta_{2}, \ldots, \zeta_{2 m}$, and then letting $z=\zeta_{k}, k=1,2, \ldots, 2 m$, in the above equation, and finally putting the obtained relations into the matrix form give us

$$
v_{n}=\left(\alpha_{n}, \beta_{n}\right) V_{n},
$$

where $\boldsymbol{\alpha}_{\boldsymbol{n}}:=\left(\alpha_{n, 1}, \alpha_{n, 2}, \ldots, \alpha_{n, m}\right), \boldsymbol{\beta}_{\boldsymbol{n}}:=\left(\beta_{n, 1}, \beta_{n, 2}, \ldots, \beta_{n, m}\right)$,

$$
\boldsymbol{v}_{n}:=\left(\frac{w_{m}^{2} \psi_{n}}{\varphi_{n}}\left(\zeta_{1}\right)-\lambda_{n} w_{m}^{2}\left(\zeta_{1}\right), \ldots, \frac{w_{m}^{2} \psi_{n}}{\varphi_{n}}\left(\zeta_{2 m}\right)-\lambda_{n} w_{m}^{2}\left(\zeta_{2 m}\right)\right)
$$

and

$$
\boldsymbol{V}_{\boldsymbol{n}}:=\left(\begin{array}{cccc}
\hat{s}_{1}\left(\zeta_{1}\right) & \hat{s}_{1}\left(\zeta_{2}\right) & \ldots & \hat{s}_{1}\left(\zeta_{2 m}\right) \\
\hat{s}_{2}\left(\zeta_{1}\right) & \hat{s}_{2}\left(\zeta_{2}\right) & \ldots & \hat{s}_{2}\left(\zeta_{2 m}\right) \\
\ldots & \ldots & & \ldots \\
\hat{s}_{m}\left(\zeta_{1}\right) & \hat{s}_{m}\left(\zeta_{2}\right) & \ldots & \hat{s}_{m}\left(\zeta_{2 m}\right) \\
\hat{t}_{1}\left(\zeta_{1}\right) & \hat{t}_{1}\left(\zeta_{2}\right) & \ldots & \hat{t}_{1}\left(\zeta_{2 m}\right) \\
\hat{t}_{2}\left(\zeta_{1}\right) & \hat{t}_{2}\left(\zeta_{2}\right) & \ldots & \hat{t}_{2}\left(\zeta_{2 m}\right) \\
\ldots & \ldots & & \ldots \\
\hat{t}_{m}\left(\zeta_{1}\right) & \hat{t}_{m}\left(\zeta_{2}\right) & \ldots & \hat{t}_{m}\left(\zeta_{2 m}\right)
\end{array}\right)
$$

with

$$
\hat{s}_{j}(z):=\frac{K_{n}\left(z, z_{j}\right)\left(\overline{z_{j}} z-1\right)}{\varphi_{n}(z) \overline{\varphi_{n}\left(z_{j}\right)}} s_{j}(z)-\frac{\varphi_{n}^{*}}{\varphi_{n}}(z)\left(\frac{\varphi_{n}}{\varphi_{n}^{*}}\right)^{\prime}\left(\frac{1}{\overline{z_{j}}}\right) t_{j}(z)
$$

and

$$
\hat{t}_{j}(z):=\frac{K_{n}\left(z, z_{j}\right)\left(\overline{z_{j}} z-1\right)}{\varphi_{n}(z) \overline{\varphi_{n}\left(z_{j}\right)}} t_{j}(z)
$$

Note the limit relations (cf. [7, Lemma 1])

$$
\lim _{n \rightarrow \infty} \frac{K_{n}\left(z, z_{j}\right)\left(\overline{z_{j}} z-1\right)}{\varphi_{n}(z) \overline{\varphi_{n}\left(z_{j}\right)}}=1
$$


for $|z|>1$, and (cf. [12, (3.10)])

$$
\lim _{n \rightarrow \infty} \frac{\varphi_{n}}{\varphi_{n}^{*}}(w)=0
$$

and consequently

$$
\lim _{n \rightarrow \infty} \frac{\varphi_{n}^{*}}{\varphi_{n}}\left(\frac{1}{\bar{w}}\right)=0 \text { and } \lim _{n \rightarrow \infty}\left(\frac{\varphi_{n}}{\varphi_{n}^{*}}\right)^{\prime}(w)=0,
$$

for $|w|<1$. So, using (29) and (30) in (27) and (28), we have

$$
\lim _{n \rightarrow \infty} \hat{s}_{j}(z)=s_{j}(z) \text { and } \lim _{n \rightarrow \infty} \hat{t}_{j}(z)=t_{j}(z) .
$$

Therefore,

$$
\lim _{n \rightarrow \infty} V_{n}=V
$$

with

$$
\boldsymbol{V}:=\left(\begin{array}{cccc}
s_{1}\left(\zeta_{1}\right) & s_{1}\left(\zeta_{2}\right) & \ldots & s_{1}\left(\zeta_{2 m}\right) \\
s_{2}\left(\zeta_{1}\right) & s_{2}\left(\zeta_{2}\right) & \ldots & s_{2}\left(\zeta_{2 m}\right) \\
\ldots & \ldots & & \ldots \\
s_{m}\left(\zeta_{1}\right) & s_{m}\left(\zeta_{2}\right) & \ldots & s_{m}\left(\zeta_{2 m}\right) \\
t_{1}\left(\zeta_{1}\right) & t_{1}\left(\zeta_{2}\right) & \ldots & t_{1}\left(\zeta_{2 m}\right) \\
t_{2}\left(\zeta_{1}\right) & t_{2}\left(\zeta_{2}\right) & \ldots & t_{2}\left(\zeta_{2 m}\right) \\
\ldots & \ldots & & \ldots \\
t_{m}\left(\zeta_{1}\right) & t_{m}\left(\zeta_{2}\right) & \ldots & t_{m}\left(\zeta_{2 m}\right)
\end{array}\right)
$$

It is easy to see that $\operatorname{det} \boldsymbol{V} \neq 0$ since $\left\{s_{j}\right\}$ and $\left\{t_{j}\right\}$ are linearly independent and form a basis of $\mathcal{P}_{2 m-1}$. Also, using Theorem 2, we have $\lim _{n \rightarrow \infty} \boldsymbol{v}_{\boldsymbol{n}}=\boldsymbol{v}$ with $\quad \boldsymbol{v}:=$

$\left(\lambda w_{m}\left(\zeta_{1}\right) w_{m}^{*}\left(\zeta_{1}\right)-\frac{\lambda}{\overline{B(0)}} w_{m}^{2}\left(\zeta_{1}\right), \ldots, \lambda w_{m}\left(\zeta_{2 m}\right) w_{m}^{*}\left(\zeta_{2 m}\right)-\frac{\lambda}{\overline{B(0)}} w_{m}^{2}\left(\zeta_{2 m}\right)\right)$.

So, letting $n \rightarrow \infty$ in $\left(\boldsymbol{\alpha}_{n}, \boldsymbol{\beta}_{\boldsymbol{n}}\right)=\boldsymbol{v}_{\boldsymbol{n}} \boldsymbol{V}_{\boldsymbol{n}}^{-1}$, we get

$$
\lim _{n \rightarrow \infty}\left(\boldsymbol{\alpha}_{n}, \boldsymbol{\beta}_{n}\right)=v V^{-1} .
$$

Now, note that $\lambda w_{m} w_{m}^{*}-\lambda w_{m}^{2} / \overline{B(0)} \in \mathcal{P}_{2 m-1}$. Thus, (24) implies

$$
\lambda w_{m}(z) w_{m}^{*}(z)-\frac{\lambda}{\overline{B(0)}} w_{m}^{2}(z)=\sum_{j=1}^{m} \alpha_{j} s_{j}(z)+\sum_{j=1}^{m} \beta_{j} t_{j}(z) .
$$


(Actually, $\alpha_{j}=0$, and $\beta_{j}=\lambda w_{m}^{\prime}\left(1 / \overline{z_{j}}\right) w_{m}^{*}\left(1 / \overline{z_{j}}\right), j=1, \ldots, m$.) Substituting $z$ by $\zeta_{k}, k=1,2, \ldots, 2 m$, we can obtain

$$
\boldsymbol{v}=\left(\alpha_{1}, \ldots, \alpha_{m}, \beta_{1}, \ldots, \beta_{m}\right) \boldsymbol{V}
$$

This and (31) give

$$
\lim _{n \rightarrow \infty}\left(\boldsymbol{\alpha}_{n}, \boldsymbol{\beta}_{n}\right)=\left(\alpha_{1}, \ldots, \alpha_{m}, \beta_{1}, \ldots, \beta_{m}\right) .
$$

Using this limit relation together with (25) and (29) we get

$$
\lim _{n \rightarrow \infty} p(z)=\frac{\lambda}{\overline{B(0)}} w_{m}^{2}(z)+\sum_{j=1}^{m} \alpha_{j} s_{j}(z)+\sum_{j=1}^{m} \beta_{j} t_{j}(z)=\lambda w_{m}(z) w_{m}^{*}(z) .
$$

This completes the proof of the corollary.

Acknoledgement. The authors thank the referee for helpful comments and suggestions.

\section{References}

[1] M. Alfaro, F. Marcellán, M.L. Rezola, and A. Ronveax, Sobolev type orthogonal polynomials: The non diagonal case, submitted,1993

[2] A. Atzmon, n-Orthonormal operator polynomials, in "Orthogonal Matrix-valued Polynomials and Applications", (Gohberg, I. Ed.), Birkhauser Verlag, Basel, 1988, 47-63.

[3] A. Cachafeiro and F. Marcellán, Orthogonal polynomials of Sobolev type on the unit circle, J. Approx. Theory, to appear.

[4] P.J. Davis, "Interpolation and Approximation", Blaisdell Publishing Co., Waltham, Mass., 1963.

[5] A.A. Gonchar, On the convergence of Padé approximants for some classes of meromorphic functions, Math. USSR Sbornik, 26 (1975), 555-575.

[6] X. Li, Representations of orthogonal polynomials for modified weight functions, submitted, 1994.

[7] X. Li and K. Pan, Asymptotic behavior of orthogonal polynomials corresponding to measure with discrete part off the unit circle, J. Approx. Theory, to appear.

[8] G. López, F. Marcellán, and W. Van Asshe, Relative assymptotics for polynomials orthogonal with repect to a discrete Sobolev inner product, Constr. Approx., to appear.

[9] G.G. Lorentz, K. Jetter, and S.D. Riemenschneider, Birkhoff Interpolation, AddisonWesley, London, 1983.

[10] F. Marcellán, M. Alfaro, and M.L. Rezola, Orthogonal polynomials on Sobolev spaces: old and new directions, J. Comp. Appl. Math., 48 (1993), 113-131.

[11] F. Marcellán and W. Van Asshe, Relative assymptotics for orthogonal polynomials with Sobolev inner product, J. Approx. Theory, 72 (1993), 193-209. 
[12] A. Máté, P. Nevai, and V. Totik, Extentions of Szegö theory of orthogonal polynomials, II, Constr. Approx., 3 (1987), 51-72.

[13] G. Pólya and G. Szegö, Problems and Theorems in Analysis II, Springer-Verlag, New York, 1976.

[14] E.A. Rakhmanov, On the asymptotics of the ratio of orthogonal polynomials, II, USSR Sbornik, 53 (1983), 105-117.

[15] G. Szegö, “Orthogonal Polynomials", 4th ed., Vol. 23, Amer. Math. Soc. Colloquium Publ., Providence, RI, 1975.

Received December 7, 1993 and revised June 24, 1994. The research of the second author was supported by Comision interministerial de Ciencia y Tecnologia of Spain (CICYT) project $\mathrm{PB}-89 / 0181 / \mathrm{C} 02 / 01$.

University of Central Florida

OrLANDo, FL 32816

E-mail address: xli@pegasus.cc.ucf.edu

AND

Departamento de IngEnieria

Escuela Politécnica Superior

Universidad Carlos III de Madrid

Avda. Mediterráneo S/N

28913 LEgANÉS, MADRID

SPAIN 


\title{
PACIFIC JOURNAL OF MATHEMATICS
}

\author{
Founded in 1951 by
}

\author{
E. F. Beckenbach (1906-1982) $\quad$ F. Wolf (1904-1989)
}

\section{EDITORS}

Sun-Yung A. Chang (Managing Editor) Robert Finn University of California

Los Angeles, CA 90095-1555

pacific@math.ucla.edu

\section{F. Michael Christ}

University of California

Los Angeles, CA 90095-1555

christ@math.ucla.edu

Nicholas Ercolani

University of Arizona

Tucson, AZ 85721

ercolani@math.arizona.edu
Stanford University

Stanford, CA 94305

finn@gauss.stanford.edu

Steven Kerckhoff

Stanford University

Stanford, CA 94305

spk@gauss.stanford.edu

Martin Scharlemann

University of California

Santa Barbara, CA 93106

mgscharl@math.ucsb.edu

\section{Gang Tian}

Massachusettes Institute of Technology

Cambridge, MA 02139

tian@math.mit.edu

\section{V.S. Varadarajan}

University of California

Los Angeles, CA 90095-1555

vsv@math.ucla.edu

Dan Voiculescu

University of California

Berkeley, CA 94720

dvv@math.berkeley.edu

\section{SUPPORTING INSTITUTIONS}

CALIF. INST. OF TECHNOLOGY CHINESE UNIV. OF HONG KONG MACQUARIE UNIVERSITY NEW MEXICO STATE UNIV. OREGON STATE UNIV. PEKING UNIVERSITY RITSUMEIKAN UNIVERSITY STANFORD UNIVERSITY TOKYO INSTITUTE OF TECHNOLOGY UNIVERSIDAD DE LOS ANDES
UNIV. OF ARIZONA

UNIV. OF BRITISH COLUMBIA

UNIV. OF CALIF., BERKELEY

UNIV. OF CALIF., DAVIS

UNIV. OF CALIF,, IRVINE UNIV. OF CALIF., LOS ANGELES

UNIV. OF CALIF., RIVERSIDE UNIV. OF CALIF., SAN DIEGO UNIV. OF CALIF., SANTA BARBARA UNIV. OF CALIF., SANTA CRUZ
UNIV. OF HAWAII

UNIV. OF MELBOURNE

UNIV. OF MONTANA

UNIV. NACIONAL AUTONOMA DE MEXICO

UNIV. OF NEVADA, RENO

UNIV. OF OREGON

UNIV. OF SOUTHERN CALIFORNIA

UNIV OF UTAH

UNIV. OF WASHINGTON

WASHINGTON STATE UNIVERSITY

The supporting Institutions listed above contribute to the cost of publication of this Journal, but they are not owners or publishers and have no responsibility for its contents or policies.

Manuscripts must be prepared in accordance with the instructions provided on the inside back cover.

The table of contents and the abstracts of the papers in the current issue, as well as other information about the Pacific Journal of Mathematics, may be found on the Internet at http://www.math.uci.edu/pjm.html.

The Pacific Journal of Mathematics (ISSN 0030-8730) is published monthly except for July and August. Regular subscription rate: $\$ 245.00$ a year (10 issues). Special rate: $\$ 123.00$ a year to individual members of supporting institutions.

Subscriptions, back issues published within the last three years and changes of subscribers address should be sent to Pacific Journal of Mathematics, P.O. Box 4163, Berkeley, CA 94704-0163, U.S.A. Prior back issues are obtainable from Kraus Periodicals Co., Route 100, Millwood, NY 10546.

The Pacific Journal of Mathematics at the University of California, c/o Department of Mathematics, 981 Evans Hall, Berkeley, CA 94720 (ISSN 0030-8730) is published monthly except for July and August. Second-class postage paid at Berkeley, CA 94704, and additional mailing offices. POSTMASTER: send address changes to Pacific Journal of Mathematics, P.O. Box 6143, Berkeley, CA 94704-0163.

PUBLISHED BY PACIFIC JOURNAL OF MATHEMATICS at University of California, Berkeley, CA 94720, A NON-PROFIT CORPORATION

This publication was typeset using AMS-LATEX,

the American Mathematical Society's TEX macro system.

Copyright (c) 1995 by Pacific Journal of Mathematics 


\title{
PACIFIC JOURNAL OF MATHEMATICS
}

\author{
Volume $175 \quad$ No. $1 \quad$ September 1996
}

Homogeneous Ricci positive 5-manifolds

Dimitri Alekseevsky, Isabel Dotti de Miatello and CARlos J.

FERRARIS

On the structure of tensor products of $\ell_{p}$-spaces

ALVARO ARIAS and JEFFREY D. FARMER

The closed geodesic problem for compact Riemannian 2-orbifolds

JosEPH E. BORZELLINO and BENJAMIN G. LORICA

Small eigenvalue variation and real rank zero

Ola Bratteli and George A. Elliott

Global analytic hypoellipticity of $\square_{b}$ on circular domains

So-CHIN CHEN

Sharing values and a problem due to C. C. Yang

XIN-Hou HuA

Commutators and invariant domains for Schrödinger propagators

Min-JEI HUANG

Chaos of continuum-wise expansive homeomorphisms and dynamical properties of sensitive maps of graphs

\section{HiS AO KATO}

Some properties of Fano manifolds that are zeros of sections in homogeneous vector bundles over Grassmannians

\section{OLIVER KÜCHLE}

On polynomials orthogonal with respect to Sobolev inner product on the unit circle

XIN Li and FRANCISCO MARCELLAN

Maximal subfields of $\mathbf{Q}(i)$-division rings

STEVEN LIEDAHL

Virtual diagonals and $n$-amenability for Banach algebras

AlAN L. T. PATERSON

Rational Pontryagin classes, local representations, and $K^{G}$-theory

Claude Schochet

An equivalence relation for codimension one foliations of 3-manifolds

SANDRA SHIELDS

A construction of Lomonosov functions and applications to the invariant subspace problem

\section{ALEKSANDER SIMONIČ}

Complete intersection subvarieties of general hypersurfaces 